\title{
ON UNITARY DILATIONS OF CONTRACTIONS
}

\section{J. J. SCHÄFFER}

Using some relatively deep facts about complex functions and spectral measures, B. Sz.-Nagy [2] has recently proved that to every contraction $A$ on a Hilbert space $H$ there corresponds a unitary operator $U$ on a larger Hilbert space $K$ so that $U^{n}$ is a dilation of $A^{n}$ for every positive integer $n$. In other words, if $\|A\| \leqq 1$, then $K$ and $U$ can be found so that $P U^{n} P=A^{n} P$, where $P$ is the projection from $K$ onto $H$. The purpose of this note is to prove the same theorem by directly exhibiting the unitary operator $U$.

Write $K$ for the direct sum of countably many copies of $H$, indexed by the set of all integers. The operator $U$ will be exhibited as a matrix whose entries $U(i, j)$ are operators on $H(i, j=0, \pm 1, \pm 2, \cdots)$. If $P$ denotes the projection from $K$ onto the zeroth coordinate space, then the fact that $P U^{n} P=A^{n} P$ will find its matricial expression in the assertion that the $(0,0)$ entry of $U^{n}$ is $A^{n}$. Let $S$ and $T$ be the positive operators on $H$ defined by $S^{2}=1-A A^{*}$ and $T^{2}=1-A^{*} A$, where 1 denotes the identity operator. The operators $U(i, j)$ are then defined as follows: $U(0,0)=A, U(-1,1)=-A^{*}, U(-1,0)=T, U(0,1)=S$, $U(i, i+1)=1$ when $i<-1$ and when $i>0$, and $U(i, j)=0$ in all other cases.

An elementary computation shows that $U$ is unitary once it is proved that $S A=A T$. This was proved by Halmos [1] by noting that $S^{2} A=A T^{2}$ and approximating the square root function in the unit interval by polynomials. The fact that $U^{n}$ is a dilation of $A^{n}$ is evident since, except for $U(0,0)$, all the nonzero entries of $U$ are above the main diagonal.

The possibility of such an explicit proof was suggested by P. R. Halmos who also contributed several useful comments.

\section{REFERENCES}

1. P. R. Halmos, Normal dilations and extensions of operators, Summa Brasiliensis Math. vol. 2 (1950) fasc. 9.

2. B. Sz.-Nagy, Sur les contractions de l'espace de Hilbert, Acta Szeged. vol. 15 (1953) pp. 87-92.

\section{UNIVERSITY OF URUGUAY}

Received by the editors July 16, 1954. 